\title{
Characterization of Tetrodes Coated with Au Nanoparticles (AuNPs) and PEDOT and Their Application to Thalamic Neural Signal Detection in vivo
}

\author{
Daae Lee ${ }^{1}$, Hyeong Cheol Moon ${ }^{2,3}$, Bao-Tram Tran ${ }^{3}$, Dae-Hyuk Kwon ${ }^{4}$, Yong Hee Kim ${ }^{5}$, \\ Sang-Don Jung ${ }^{5}$, Jong Hoon Joo $^{1}$ and Young Seok Park ${ }^{2,3 *}$ \\ ${ }^{1}$ Department of Advanced Materials Engineering, Chungbuk National University, Cheongju $28644,{ }^{2}$ Department of \\ Neurosurgery, Chungbuk National University Hospital, Cheongju 28644, ${ }^{3}$ Department of Neurosurgery, Chungbuk National \\ University, Cheongju 28644, ${ }^{4}$ Neuroscience Research Institute, Brain-Bio Center, University of Suwon, Hwaseong 18323, \\ ${ }^{5}$ Synaptic Devices Research Section, Electronics and Telecommunications Research Institute, Daejeon 34129, Korea
}

\begin{abstract}
Tetrodes, consisting of four twisted micro-wires can simultaneously record the number of neurons in the brain. To improve the quality of neuronal activity detection, the tetrode tips should be modified to increase the surface area and lower the impedance properties. In this study, tetrode tips were modified by the electrodeposition of Au nanoparticles (AuNPs) and dextran (Dex) doped poly (3,4-ethylenedioxythiophene) (PEDOT). The electrochemical properties were measured using electrochemical impedance spectroscopy (EIS) and cyclic voltammetry (CV). A decrease in the impedance value from $4.3 \mathrm{M} \Omega$ to $13 \mathrm{k} \Omega$ at $1 \mathrm{kHz}$ was achieved by the modified tetrodes. The cathodic charge storage capacity $\left(\mathrm{CSC}_{\mathrm{C}}\right)$ of AuNPs-PEDOT deposited tetrodes was $4.5 \mathrm{mC} / \mathrm{cm}^{2}$, as determined by CV measurements. The tetrodes that were electroplated with AuNPs and PEDOT exhibited an increased surface area, which reduced the tetrode impedance. In vivo recording in the ventral posterior medial (VPM) nucleus of the thalamus was performed to investigate the single-unit activity in normal rats. To evaluate the recording performance of modified tetrodes, spontaneous spike signals were recorded. The values of the L-ratio, isolation distance and signal-to-noise (SNR) confirmed that electroplating the tetrode surface with AuNPs and PEDOT improved the recording performance, and these parameters could be used to effectively quantify the spikes of each cluster.
\end{abstract}

Key words: microelectrodes, electrodes, Implanted, neuron, Signal detection, Thalamus

Received July 2, 2018, Revised November 12, 2018, Accepted November 16, 2018

* To whom correspondence should be addressed. TEL: 82-43-269-6080, FAX: 82-43-273-1614 e-mail:youngseokparkmd@gmail.com

\section{INTRODUCTION}

Extracellular recording focuses on detecting large-scale neuronal activity as the électric signal code' in vivo $[1,2]$. Tetrodes consisting of four twisted microwires have proved to be valuable tools for the simultaneous recording of multiple neurons because they can isolate many single neurons simultaneously [3], Tetrode charac- 
terization depends on the tetrode materials, diameters and surface area. Various microelectrode materials have been reported such as tungsten, titanium and gold, which are known to be biocompatible materials [4-6]. Many attempts to reduce the electrode size to minimize tissue damage and obtain a high neural network spatial resolution have been reported $[1,7,8]$. Although small electrodes enable avoiding tissue damage and provide high selectivity for a neural recording, the impedance value is high, which reduces the quality of a recording.

In general, a high impedance leads to a lower signal-to-noise ratio (SNR) of a recording, which reduces the performance of a neuronal signal recording, making it difficult to detect low-level neural signals. Undifferentiated background action potentials can contribute neural noise, and this noise can add to the electrode impedance, which is inversely proportional to the surface area [9, 10]. To address these issues, low-impedance tetrodes are desirable; increasing the surface area of a tetrode via surface modification with nanoparticles can contribute to reducing the impedance. Many attempts have been made to modify the surfaces of neural electrodes via electrochemical deposition, sputter deposition and spray deposition $[11,12]$. Electroplating, which occurs via electrochemical deposition, is a relatively conventional method because no additional patterning process is performed, such as in sputter deposition.

Surface modification via electroplating causes the growth of crystallites on tetrode tips. These crystallites can form nanostructures on the surface that increase the effective surface area of a tetrode. Furthermore, surface modification plays an important role in improving the charge transfer properties at the electrode and tissue interface. Various materials have been applied to modify the surfaces of electrodes, including gold, platinum $(\mathrm{Pt})$ black, iridium oxide ( $\mathrm{IrOx}$ ), carbon nanotubes (CNTs) and many kinds of conductive polymers (CPs) [8, 13-15]. Previous studies involving the coating of materials onto tetrodes to lower their impedance values and enlarge their effective surface areas have been conducted $[8$, $16,17]$.

Au nanoparticles (AuNPs) are well-known materials that are non-toxic, highly conductive and biocompatible [18]. AuNPs are widely used in the field of biosensors, multielectrode arrays (MEAs), and microelectrodes [19-21]. MEAs with electrodeposited AuNPs have been reported to possess increased surface area and improved recording performance in vitro [19]. Electron beam lithography, physical deposition and electrochemical deposition are among the many methods used to attach gold to surfaces. In this study, AuNPs were attached to the surfaces of tetrodes via the electrochemical deposition method, which is a simple method used to fabricate tetrode tips.
CPs such as polypyrrole (PPy), polythiophene (PTh) and poly(3,4-ethylenedioxythiophene) (PEDOT) have been commonly applied the over coating materials of neural electrodes since the size of electrodes has decreased [22-27]. The application of CPs has many advantages, as they exhibit fast and high-charge delivery capacities due to their excellent intrinsic conductivity and provide a large surface area when used as coating materials [8, 23, 28-31]. CPs effectively increase the surface area of the electrodes, without changing the geometric surface area, and lower the impedance [31-33]. Among these CPs, poly(3,4-ethylenedioxythiophene) (PEDOT) was reported as a biocompatible material in an in-vivo polymerization study $[31,34]$. The morphology of PEDOT displays a microscale cauliflower-like structure that can effectively increase the surface area and decrease the impedance of the electrodes [35]. Furthermore, PEDOT coatings enable the fabrication of ultrasmall, high-channel-count arrays for a constant interface with the brain [33]. PEDOT has beneficial properties such as electrical, chemical and environmental stability and offer better conductivity and thermal stability than those of PPy [36,37].

The purpose of our study was to investigate the improved recording quality of tetrodes achieved by altering their electrochemical properties via surface modifications though the electroplating method, which is a simple method used to fabricate tetrode tips. The effect of the electrochemical deposition of AuNPs and PEDOT on tetrodes was shown to reduce the electrode impedance. Although some research on the effects of AuNPs and PEDOT as separate coating materials has been carried out, no studies involving the sequential electrodeposition of PEDOT and AuNPs onto tetrodes have been reported. This paper attempts to show the possibility of further reducing the impedance of recording sites and increasing the charge storage capacity of tetrodes via this double coating. Furthermore, to elucidate the improvement in the recording quality, the modified tetrodes were used to record neural signals in the ventral posterior medial (VPM) nucleus of Sprague Dawley (S-D) rats. The L-ratio, isolation distance (ID) and signal-to-noise (SNR) were calculated. These parameters can be used to quantify the spikes of each cluster. The morphology of the electroplated tetrodes was confirmed via scanning electron microscopy (SEM), and the electrochemical properties were determined via electrochemical impedance spectroscopy (EIS) and cyclic voltammetry (CV) measurements.

\section{MATERIALS AND METHODS}

\section{Fabrication of tetrodes}

Tetrode bundles were fabricated according to the standard methods [16, 38, 39]. Polyimide-insulated $13-\mu \mathrm{m}$ nickel-chromium 
wires (California Fine Wire, Grover Beach, CA) were used as the tetrode. To obtain a rigid bundle, twisted wires were heated with a heat gun (GHG 500-2, Bosch, Romania) at $300^{\circ} \mathrm{C}$ using a low flow for $1 \mathrm{~min}$ with different angles. The tetrodes were carefully cut using sharp micro scissors (14054-13, Fine Science Tools, Foster City, CA). To achieve a consistent surface area cross-section, the fabricated tetrode wires were cut vertically, and the sizes of the bare electrodes were confirmed based on the cross-section of the SEM image. The manufactured tetrode wires were connected to a 32-channel electronic interface board (EIB), and each wire was fixed with a gold pin.

\section{Electrochemical-deposition of AuNPs and PEDOT}

AuNPs were deposited onto the exposed tips of the polyimideinsulated $13-\mu \mathrm{m}$ nickel-chromium wires from a stimulus isolator (A365D, World Precision Instruments, Sarasota, FL). The AuNP electroplating solution consisted of a non-cyanide, gold-plating solution (Neuralynx Inc., Bozeman, MT, USA) mixed with a PEG solution $(1 \mathrm{mg} / \mathrm{mL}$ in DI water, Sigma-Aldrich, St Louis, MO), which acted as an inhibitor during the electrodeposition process and enlarged the surface area [40]. The current used for electroplating was $200 \mu \mathrm{A}$, which was applied for $60 \mathrm{~s}$ to avoid wire shorting.

The AuNPs-coated tetrode tips were further coated with dextran (Dex)-doped PEDOT. PEDOT-Dex was deposited from a solution consisting of a mixture of $0.02 \mathrm{M}$ ethylene dioxythiophene (EDOT) and $2 \mathrm{mg} / \mathrm{ml}$ Dextran (Dex). Electrochemical deposition (with $\mathrm{CV}$ ) was performed for 5 cycles from $0 \mathrm{~V}$ to $1 \mathrm{~V}$ at scan rate of $20 \mathrm{mV} / \mathrm{s}$.

\section{Electrochemical and physical characterization of the elec- trodes}

EIS was performed using the ModuLab Femtostat system (Solartron Analytical, Berks, UK), and measurements were carried out with a three-electrode electrochemical cell in a $0.1 \mathrm{M} \mathrm{KCl}$ solution $\left(1.0 \mathrm{mM} \mathrm{K}_{4} \mathrm{Fe}(\mathrm{CN})_{6} / \mathrm{K}_{3} \mathrm{Fe}(\mathrm{CN})_{6}\right)$. The tetrodes, an $\mathrm{Ag} / \mathrm{AgCl}$ electrode in a saturated $\mathrm{KCl}$ solution and a platinum wire were used as the working, counter and reference electrodes, respectively. EIS was carried out at an amplitude of $200 \mathrm{mV}$, and the frequency range was between $0.1 \mathrm{~Hz}$ and $100 \mathrm{kHz}$.

$\mathrm{CV}$ analyses were carried out in the basic three-electrode cell with the same configuration as that of the EIS system. CV was performed at a scan rate of $50 \mathrm{mV} / \mathrm{s}$, from $-0.1 \mathrm{~V}$ to $0.8 \mathrm{~V}$ (vs. $\mathrm{Ag} /$ $\mathrm{AgCl}$ ), using a potentiostat (Solatron Analytical, ModuLab, Berks, $\mathrm{UK}$ ) for bare tetrodes ( $\mathrm{NiCr}$ ), the AuNPs-coated tetrodes. In the case of PEDOT-deposited tetrodes, $\mathrm{CV}$ was conducted from - 0.8 $\mathrm{V}$ to $0.7 \mathrm{~V}$. The $\mathrm{CV}$ measurements and EIS were performed before and after electroplating was performed.
The microstructure of each tetrode surface was evaluated using SEM to determine the morphology of the AuNPs and PEDOT. The electroplated surface was studied via EIS and CV measurements instead of by conducting an additional microstructural analysis.

\section{Surgical implantation and in vivo recording}

Animal experiment protocols were approved according to the National Institute of Health guidelines at Chungbuk National University (CBNUR-1072-17). Female adult SD rats (200 220 g; Koatech, Pyeongtaek, Korea) were maintained under standard housing conditions (12-hour light/dark cycle) and were allowed to drink water and eat food pellets ad libitum. The animals were randomly divided into three groups: (1) the NiCr group ( $n=3)$; (2) the AuNPs group ( $n=3)$; and (3) the PEDOT group ( $n=3)$. Each group was randomly separated from the pool of animals in all cages. All rats were anesthetized with a mixture of $15 \mathrm{mg} / \mathrm{kg}$ letamine/zolazepam (Zoletil50 ${ }^{\circledR}$; Virbac Laboratories, Carros, France) and 9 mg/ kg xylazine (Rompun ${ }^{\circledR}$; Bayer, Seoul, South Korea) in saline solution. To target the ventral posteromedial nucleus of the thalamus (VPM) [coordinates: anterior-posterior (AP), -3.5 mm; mediallateral (ML), -2.8 mm, dorsal-ventral (DV), -6.0 $\mathrm{mm}$ from the dura] in the skull, the bone was drilled to expose the underlying dura mater.

The tetrodes (diameter: $13 \mu \mathrm{m}$ ) were implanted into the VPM nuclei of the rats. Surgical procedures were conducted with a surgical microscope (Wesco SWF 10X/22, Illinois, USA) for visual guidance. The recorded neuronal activity was displayed by a Cheetah acquisition system (Neuralynx Inc., Bozeman, MT, USA), which obtained samples at 30,303 Hz. Spikes collected using the Cheetah system were identified as single-unit activity with a standard waveform-shaped and inter-spike-interval histogram obtained using the SpikeSorter3D software (Neuralynx Inc., Bozeman, MT, USA). The signals were digitized and recorded with bandpass from $900 \mathrm{~Hz}$ to $6000 \mathrm{~Hz}$. And threshold level set was 50 $\mu \mathrm{V}$. The single units of neuronal activity were analyzed with respect to an overall firing rate according to each tetrode.

The L-ratio and Isolation distance (ID) were calculated to quantify the quality of a cluster consisting of recorded spikes, which can calculate how well one cluster is separated from other clusters recorded on the same electrode and these values were automatically calculated after spike sorting by Harris et al. and SchmitzerTorbert et al. [41, 42]. From the Mahalanobis distance, the following equation was used to measure spike $i$ from the center of the cluster C: $D_{i, c}^{2}=\left(x_{i}-\mu_{c}\right)^{\mathrm{T}} \Sigma_{c}^{-1}\left(x_{i}-\mu_{c}\right)$ where $\mathrm{D}$ denotes the distance from each event $\left(\mathrm{x}_{\mathrm{i}}\right)$ to the mean feature vector $\left(\mu_{\mathrm{i}}\right)$, and $\Sigma_{\mathrm{c}}$ represents the covariance matrix $\left(\sum_{c}=X^{T} X\right)$ in the elected cluster. Unlike Euclidean 
distance, the Mahalanobis distance reflects the elliptical shape of the cluster, which is the result of multivariate properties. When all points are surrounded by the same direction, the Mahalanobis distance equals to the Euclidean distance.

The L-ratio is defined as $\mathrm{L}(\mathrm{C})=\sum_{\mathrm{i} \in \mathrm{C}} 1-\mathrm{CDF}_{\mathrm{x}_{\mathrm{dat}}}\left(\mathrm{D}_{\mathrm{i}, \mathrm{C}}^{2}\right)$, where CD denotes the cumulative probability value of $\mathrm{x}^{2}$. When the distribution of a cluster is Gaussian, the $\mathrm{D}^{2}$ value for the spikes of the cluster is equal to the square of 8 degrees of freedom because the tetrode has four electrodes with two dimensions. L-ratio represent how well the spike are separated from the other spikes that are recorded on the same tetrodes. From $L_{\text {ratio }}(c)=\frac{L(c)}{n_{c}}$, the L-ratio can be calculated, where $\mathrm{n}_{\mathrm{C}}$ is the number of the cluster $\mathrm{C}$. When cluster $\mathrm{C}$ contains $\mathrm{n}_{\mathrm{C}}$ cluster spikes; the Isolation distance (ID) represents the $\mathrm{D}_{2}$ value of the $\mathrm{n}_{\mathrm{C}}^{\text {th }}$ nearest noise spike and ID values can represent how distant the cluster spikes are from the other spike and identify well-seperated clusters. When the ID values are high and the L-ratio values are low, the cluster could be well separated.

To estimate the signal-to-noise ratio (SNR) and the spike peakto-peak amplitude, the root mean square (RMS) noise of the background noise (RMS noise), all analysis was carried out on continuous recordings (NCS data) using MATLAB (MathWorks, Natick, MA), and the FieldTrip toolkit (Donders Institute for Brain, Cognition and Behaviour, Nijmegen, Netherlands) was used, providing pure MATLAB implementations to load Neuralynx format directly. Neural recording segments were analyzed offline to perform the spike detection and sorting using the MATLAB-based toolkit, Wave_clus $[43,44]$. An amplitude threshold was automatically set to $5 \sigma_{n}$ where $\sigma_{n}$ is an estimate of the RMS noise. For each detected spike, 64 samples (i.e., $2.5 \mathrm{~ms}$ ) were stored for later analysis. After that, the signal amplitude and SNR were estimated using customautomated MATLAB software. The signal amplitude for a cluster was defined as the mean peak-to-peak amplitude of the detected spike waveforms. The SNR was determined as the ratio of the spike (peak-to-peak) to noise ( $2 \times \mathrm{RMS}$ noise) $[33,45]$. For 9-tetrode dataset (3-tetrode dataset per group), the SNR values are calculated separately for each of the 4 electrodes, then the final SNR values are the average of the 4 per-electrode SNR values.

\section{Statistics}

Data from the in vivo recordings and impedance analysis represented in the following form: mean \pm standard deviation (SD). We performed one-way analysis of variance (ANOVA) using GraphPad Prism (GraphPad Software, San Diego, CA, USA), with the data being presented as an in vivo recording. For all tests, the data are presented as the mean $\pm \mathrm{SD},{ }^{\star} \mathrm{p}<0.01$.

\section{RESULTS}

\section{Electrochemical and physical properties of the tetrodes}

Fig. 1 shows SEM images of the unmodified bare tetrodes ( $\mathrm{NiCr}$ ),

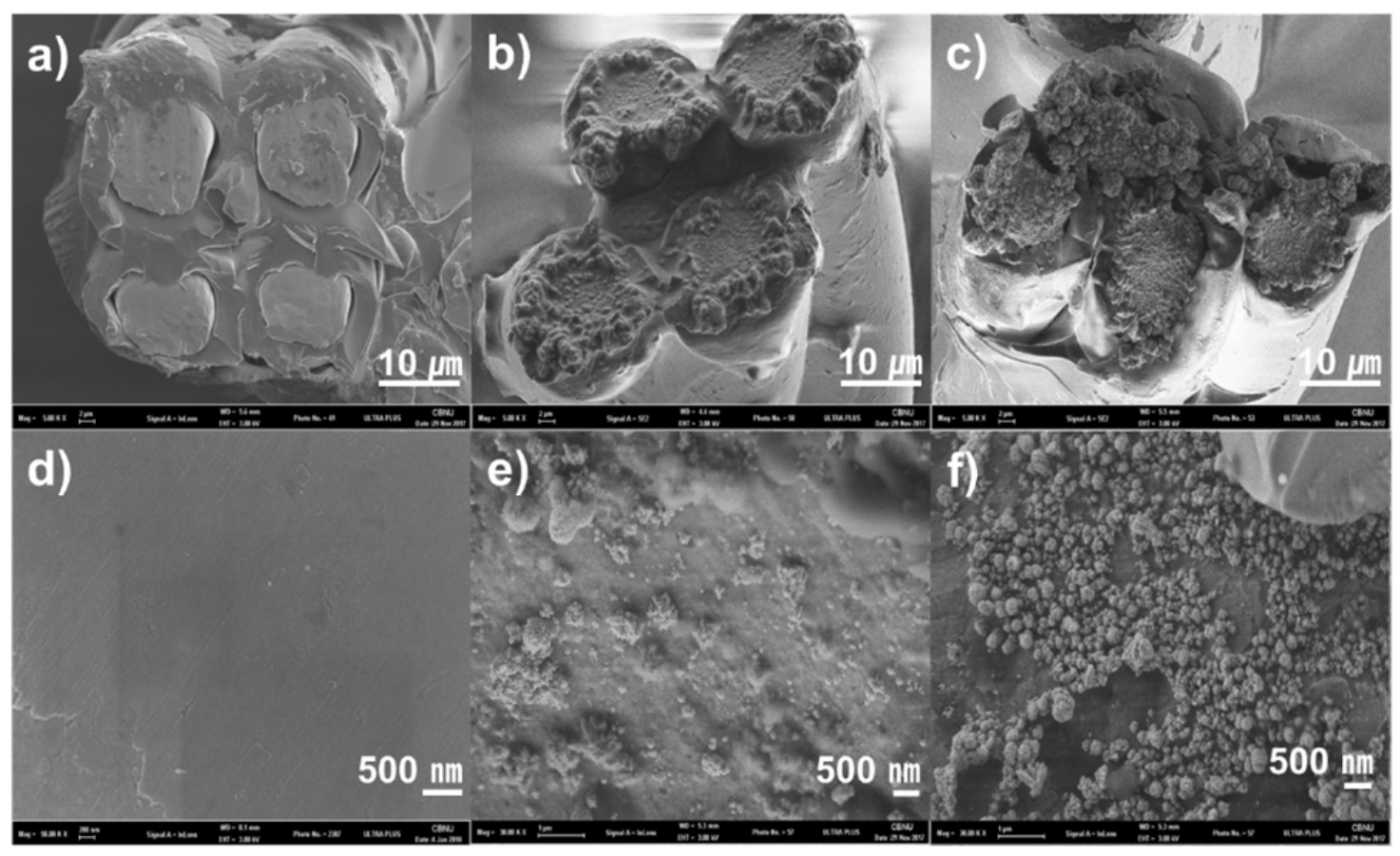

Fig. 1. a) SEM images of a bare NiCr tetrode without surface modification; b) AuNPs-coated tetrode; c) AuNPs-PEDOT-coated tetrode; d) f) magnified image of a) NiCr, b) AuNPs and c) AuNPs and PEDOT, respectively. 
the AuNPs-deposited tetrodes and the AuNPs-PEDOT-coated tetrodes, respectively. As shown in Fig. 1a), the NiCr electrode had a flat surface. Two-dimensional surfaces are exposed around the tetrode tips, and the nonexposed wires are insulated with polyimide. After the AuNPs were electroplated on the surface, they were distributed in an irregular arrangement on the $\mathrm{NiCr}$ electrode. As shown in Fig. 1c), the SEM images demonstrated that the PEDOT coating was well-plated to the AuNPs-coated surface; thus, the tips of the tetrodes had a high surface area due to the resultant nanostructures, which can greatly decrease the impedance of the electrode recording site.

The impedance characterization reflects the electrical and electrochemical properties of the tetrodes. The impedance value changes as a result of the applied materials, the electrolyte solution and the surface morphology. High impedance values are expected to contribute signal noise with a low signal-to-noise ratio which could apply a large electrode potential to the tissue due to undesirable electrochemical reactions. Therefore, a low impedance value is important for recording of microscopic neural activity. By electrodepositing AuNPs and PEDOT, the surface roughness was increased, and the impedance of the electrode sites was reduced. The electrochemical impedance was measured for the bare $\mathrm{NiCr}$ wires and the two modified tetrodes from $0.1 \mathrm{~Hz}$ to $100 \mathrm{kHz}$ (Fig. 2). The impedance of the bare NiCr wires was approximately 4.3 $\mathrm{M} \Omega$ at $1 \mathrm{kHz}$. The impedance of the AuNPs-coated tetrodes decreased to $306 \mathrm{~K} \Omega$, indicating that the AuNPs could effectively decrease the impedance and increase the surface area of the tetrode, as shown in Fig. 1. When PEDOT was additionally deposited onto the AuNPs-coated tetrodes, the impedance further decreased to $13 \mathrm{k} \Omega$. Electroplating both AuNPs and PEDOT onto the surface was found to decrease the impedance values of the tetrodes at 1 $\mathrm{kHz}$ to a statistically significant degree $(\mathrm{p}=0.0069, \mathrm{p}=0.0002)$ compared with the bare $\mathrm{NiCr}$ wire in the $0.1 \mathrm{M} \mathrm{KCl}$ solution $(1.0 \mathrm{mM}$ $\left.\mathrm{K}_{4} \mathrm{Fe}(\mathrm{CN})_{6} / \mathrm{K}_{3} \mathrm{Fe}(\mathrm{CN})_{6}\right)$. The reason for comparing the impedance at $1 \mathrm{kHz}$ is that neural activity occurs at approximately 1-300 $\mathrm{kHz}$. The bode plots from Fig. 2a) also show that the impedance of bare $\mathrm{NiCr}$ wire increased with a decrease in frequency, while the PEDOT-coated tetrodes acted as capacitors at low frequency $(<100 \mathrm{~Hz})$ and as resistors at high frequency $(>100 \mathrm{~Hz})$. The resistive interface at low frequency indicates that the charge transfer during signal recording is due to a reversible faradaic reaction. The results indicated that the impedance of the modified tetrodes was reduced, due to the increased surface area as a result of the electrodeposition of AuNPs and PEDOT. Furthermore, the high electronic conductivity $(300>\mathrm{S} / \mathrm{cm})$ and redox reaction resulting from the use of PEDOT contribute to reduction in the impedance value, which can be deduced through Nyquist plot and CV measurements mentioned below.

EIS, which shows the electrical and electrochemical properties of the tetrodes, is used to characterize the impedance. In this study, the equivalent circuit model used for the tetrodes is adapted from the theoretical models representing the electrode-electrolyte impedance [46]. Fig. 3a) represents Equivalent circuit model R(RQ) for AuNPs- and PEDOT-electroplated tetrodes. Using the ModuLab Femtostat system, the $R\left(R_{Q}\right)$ model represents a circuit in which a solution resistance $\left(R_{S}\right)$ is in series with the constant phase angle element (CPE) and a charge transfer resistance $\left(R_{t}\right)$ connected in parallel. $R_{S}$ refers to the resistance measured between the working electrode and the reference electrode. CPE represents the double-layer capacitance of the recording surface and $\mathrm{R}_{\mathrm{t}}$ is directly
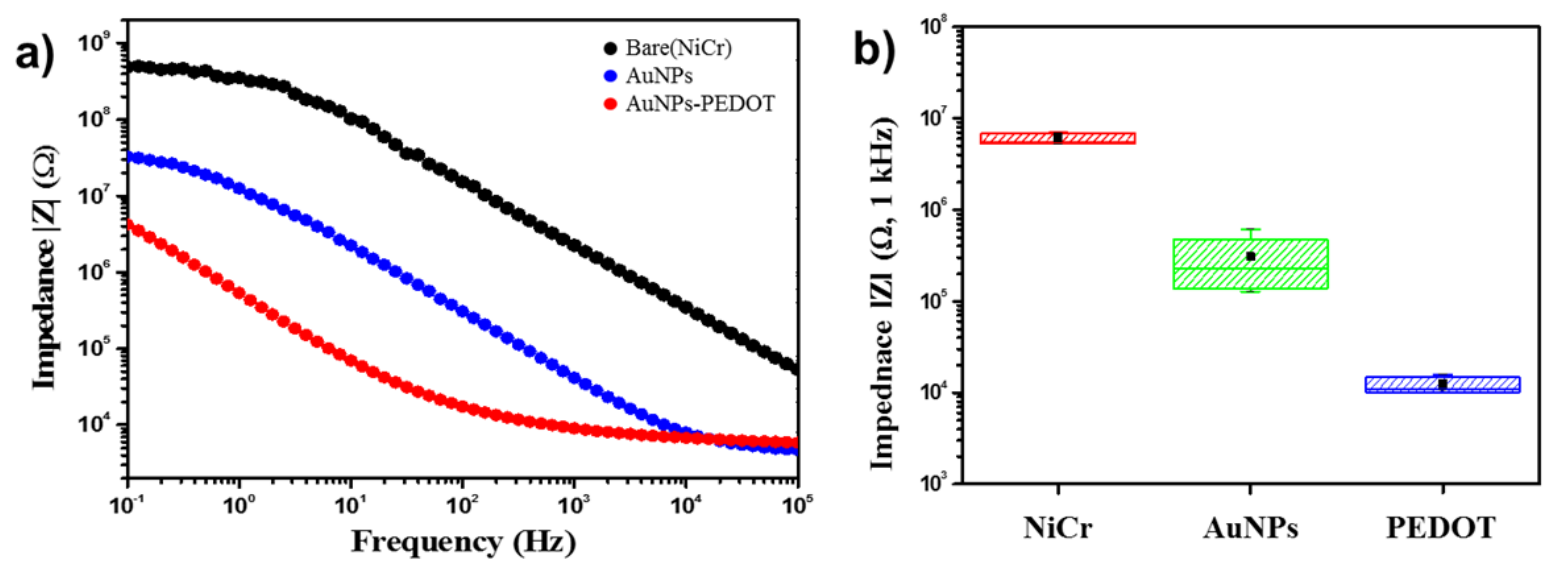

Fig. 2. a) Bode plots of NiCr, AuNPs-coated and AuNPs-PEDOT-coated tetrodes. The impedance value, as a function of frequency, obtained via EIS shows that the AuNPs- and AuNPs-PEDOT-coated tetrodes present low impedance values. b) Average in vitro impedance of NiCr ( $\mathrm{n}=15)$, AuNPs ( $\mathrm{n}=18$ ) and PEDOT $(\mathrm{n}=15)$ at $1 \mathrm{kHz}$. The AuNPs- and AuNPs-PEDOT-coated tetrodes exhihit significantly lower impedance values than the bare NiCr tetrodes due to the expanded surface area resulting from electrodeposition. All data in b) are presented as the mean \pm SD. 

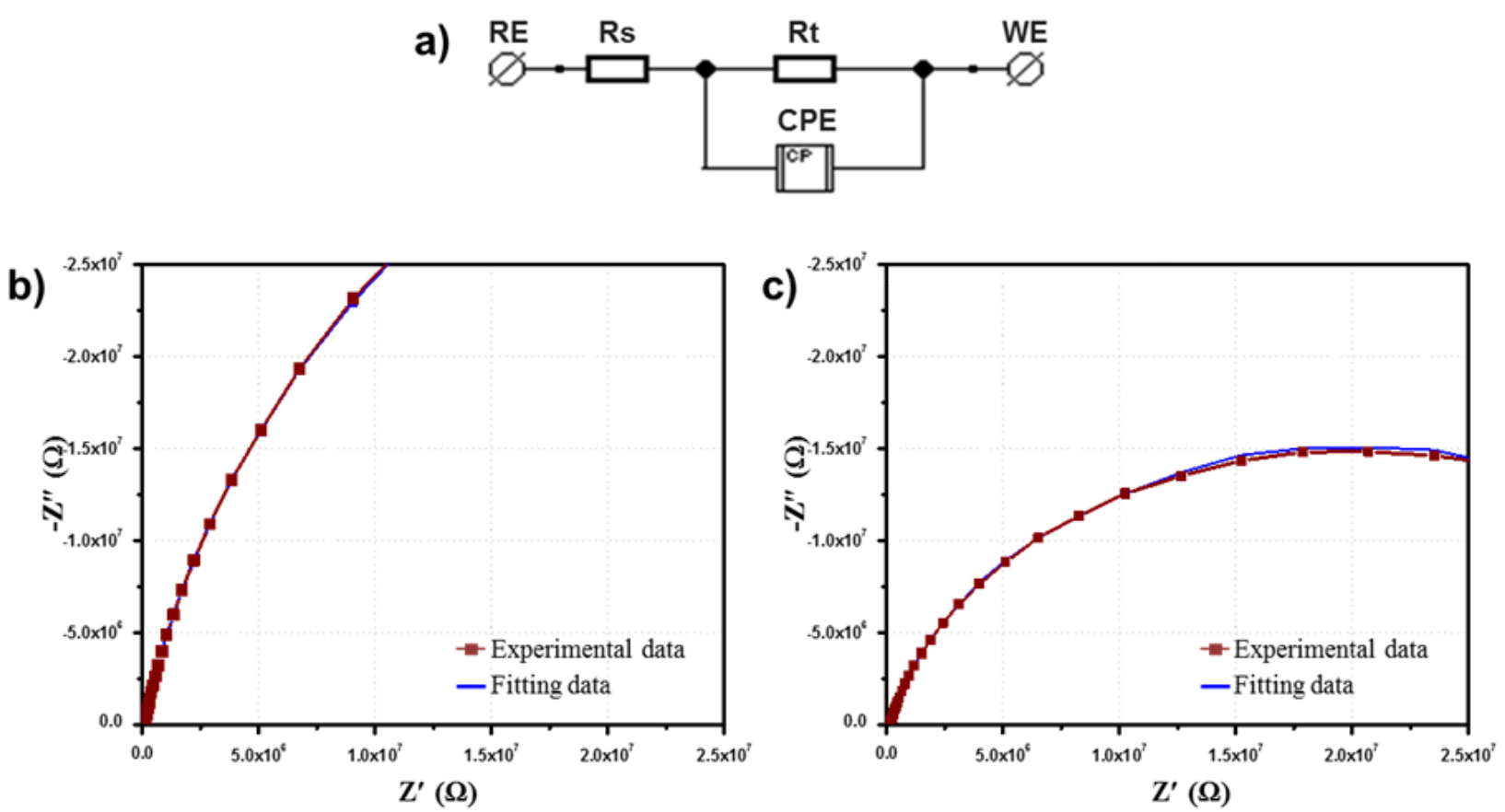

Fig. 3. a) Equivalent circuit model R(RQ) for AuNPs- and PEDOT-electroplated tetrodes. Rs is the solution resistance, Rt is a charge transfer resistance from the electrodeposited materials. CPE is the constant phase angle element. b) Fitting EIS data and experimental EIS data of AuNPs-coated tetrode; $\mathrm{c}$ ) Fitting EIS data and experimental EIS data of the PEDOT-coated tetrodes. These fitting results were matched with the experimental results well (Modulab).

Table 1. Fitted impedance values for the equivalent circuit model R(RQ) for AuNPs- and PEDOT-electroplated tetrodes

\begin{tabular}{lcccc}
\hline & $\mathbf{R}_{\mathbf{s}}(\mathbf{k} \boldsymbol{\Omega})$ & $\mathbf{C P E}(\boldsymbol{\mu} \mathbf{F})$ & $\boldsymbol{\alpha}$ & $\mathbf{R}_{\mathbf{t}}(\mathbf{M} \boldsymbol{\Omega})$ \\
\hline AuNPs & 43.7 & 1.44 & 0.85 & 138.5 \\
AuNPs-PEDOT & 56.5 & 5.69 & 0.78 & 43.8 \\
\hline
\end{tabular}

related to the charge transfer resistance [47]. Table 1 shows the fitted impedance values. According to the equivalent circuit model, the goodness of fit of each sample estimated from the $\mathrm{x}$-square test was $1.1 \times 10^{-3}$ and $5.6 \times 10^{-3}$, respectively. It is quite good agreement with the curves generated from the fitted results, as shown in Fig. 3b-c). From the $\mathrm{R}_{\mathrm{t}}$ values indicated by the fitted results, the PEDOT-coated tetrodes exhibit low resistance, which is related to the conductivity of the material and redox reaction; this value is reasonable considering the EIS measurements.

\section{CV and Charge storage capacity}

$\mathrm{CV}$ can confirm the reversibility of electrochemical reactions and the quantity of electroactive material on an electrode. The CV results obtained for the NiCr, AuNPs-coated and AuNPs-PEDOTcoated tetrodes are compared in Fig. 4. The AuNPs-PEDOTcoated tetrode exhibited a large hysteresis compared with the $\mathrm{NiCr}$ and AuNPs-coated tetrodes, as shown in Fig. 4, indicating that the modified tetrodes undergo an active electrochemical reaction. CV typically measures the characteristics of a redox reaction and the charge storage capacity of electrodes. The cathodic charge storage capacity $\left(\mathrm{CSC}_{\mathrm{C}}\right)$, which represents the improvement in capacitance of the electrode, was calculated by integrating the cathodic currents of a CV cycle. As shown in Fig. 4, the bare NiCr, AuNPscoated and AuNPs-PEDOT-coated tetrodes were tested to study their electrochemical behavior. The $\mathrm{CSC}_{\mathrm{C}}$ for $\mathrm{NiCr}$ was $0.084 \mathrm{mC} /$ $\mathrm{cm}^{2}$. After electrodeposition of the AuNPs and AuNPs-PEDOT, the $\mathrm{CSC}_{\mathrm{C}}$ of the tetrodes dramatically increased to $0.448 \mathrm{mC} / \mathrm{cm}^{2}$ and $4.5 \mathrm{mC} / \mathrm{cm}^{2}$, respectively. It was demonstrated that the $\mathrm{CSC}_{\mathrm{C}}$ of the AuNPs-PEDOT-coated tetrode was much higher than that of the bare NiCr and AuNP-coated tetrodes, which indicated that PEDOT provided a large surface area on the tetrode tip.

\section{In vivo recording}

To investigate the neural recording ability of each tetrode, three different tetrodes (bare, AuNPs-coated and AuNPs-PEDOT-coated) were implanted in the thalamic VPM nuclei of S-D rats, and spontaneous neural signals were recorded. Fig. 5a), b) shows the corresponding spike feature space and spike waveforms at each of four wires and unclustered spikes were not shown. The horizontal axis represents time (ms), and the vertical axis represents voltage $(\mu V)$. To measure the recording quality of the tetrode, SNR values 

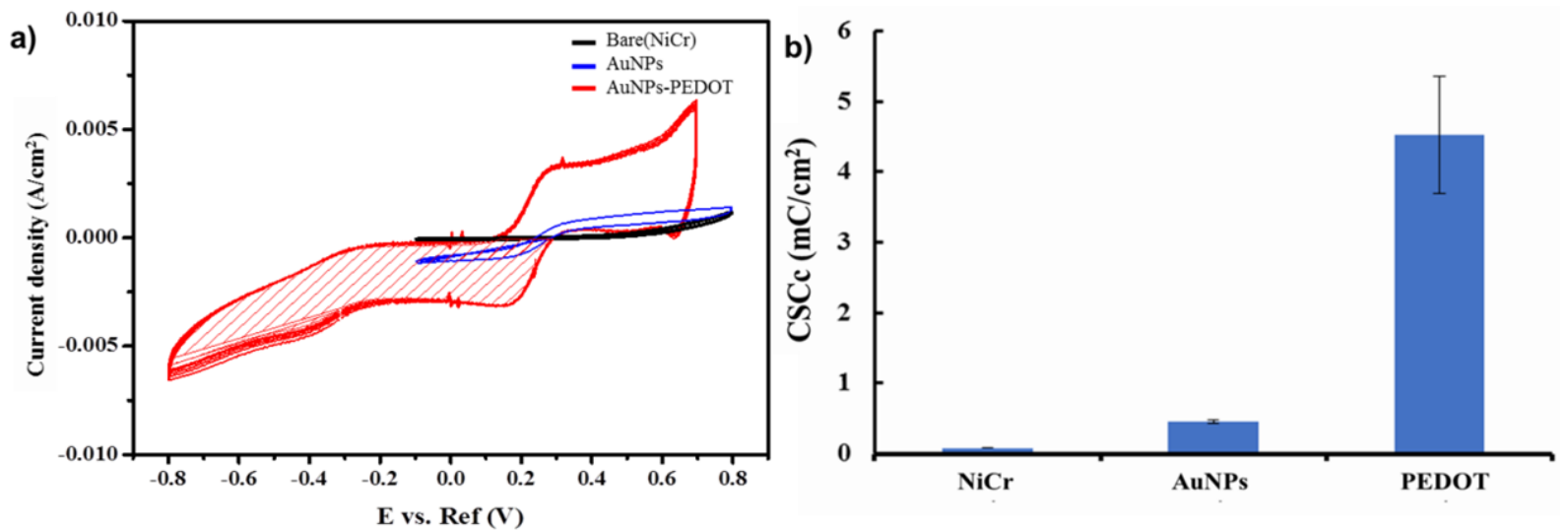

Fig. 4. a) Cyclic voltammetry measurements of the bare NiCr tetrode, AuNPs-coated tetrode and AuNPs-PEDOT-coated tetrode in $0.1 \mathrm{M} \mathrm{KCl}$ solution $\left(1.0 \mathrm{mM} \mathrm{K}_{4} \mathrm{Fe}(\mathrm{CN})_{6} / \mathrm{K}_{3} \mathrm{Fe}(\mathrm{CN})_{6}\right)$ between $-0.1 \mathrm{~V}$ and $0.8 \mathrm{~V}$ and between $0.8 \mathrm{~V}$ and $0.7 \mathrm{~V}$ (vs. the reference electrode) at a scan rate of $50 \mathrm{mV} / \mathrm{s}$. b) Average of the cathodic charge storage capacity $\left(\mathrm{CSC}_{\mathrm{C}}\right)$ of each tetrode, bare $\mathrm{NiCr}(\mathrm{n}=6)$, AuNPs-coated $(\mathrm{n}=6)$ and AuNPs-PEDOT-coated ( $\mathrm{n}=11$ ).

\section{Spike feature space}

a)

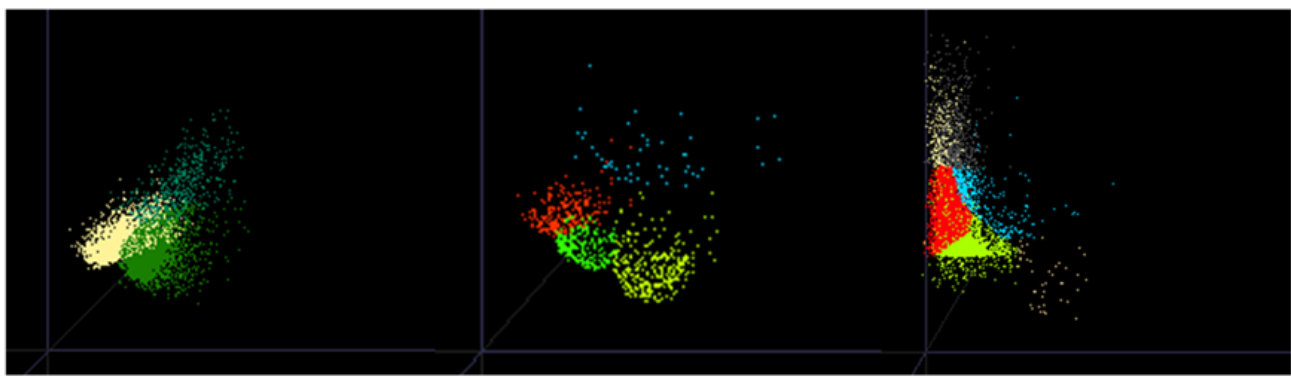

$\mathrm{NiCr}$

AuNPs

AuNPs-PEDOT

Spike waveforms

b)

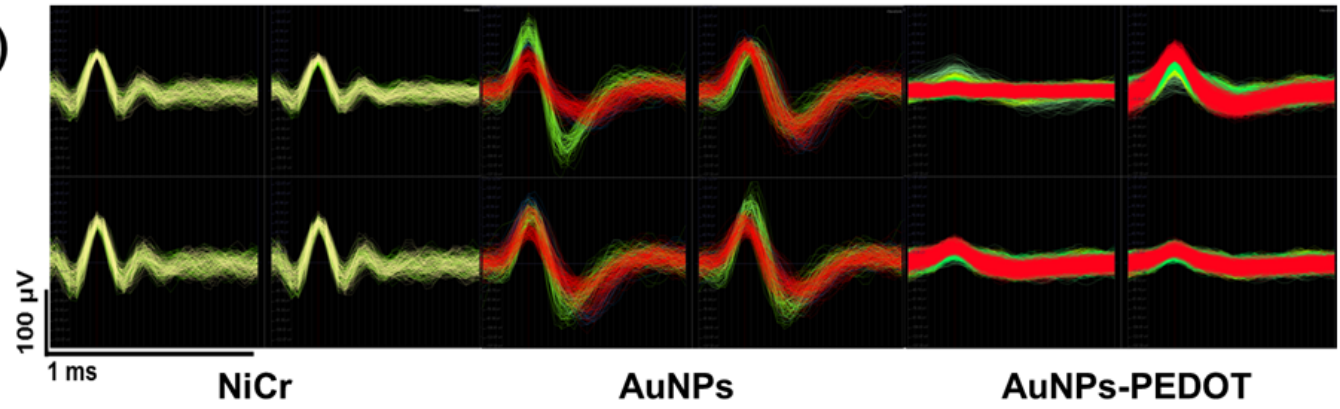

Fig. 5. a) Spike feature space from the result of in vivo recording of the tetrodes (NiCr, AuNPs-coated, and AuNPs-PEDOT-coated respectively) recorded using the SpikeSorter3D software. The different colors of the waveforms indicate the distinct clusters resulting from spike sorting. b) Spike waveforms of action potentials on the tetrodes (NiCr, AuNPs-coated, and AuNPs-PEDOT-coated).

were calculated. The average (AVE) and standard deviation (STD) of the SNR for each tetrode (NiCr, AuNPs-, AuNPs-PEDOT) were $8.72 \pm 0.03 \mathrm{~dB}, 8.90 \pm 0.25 \mathrm{~dB}$ and $9.02 \pm 0.59 \mathrm{~dB}$, respectively.

According to the results of in vivo recording, the L-ratio and ID values were calculated, which quantitatively indicate the number of distinguished signals from the recording data [41]. Fig. 6a) shows ID the values of the sorted spikes and the spontaneous spike from the bare NiCr, AuNPs-coated and AuNPs-PEDOTcoated tetrodes. As shown in Fig. 6b), the L-ratio of the PEDOTelectrodeposited tetrodes was significantly lower than that of the bare NiCr tetrodes. The comparison of the L-ratio and ID values among the bare and electroplated tetrodes is shown in Table 2. 
a)

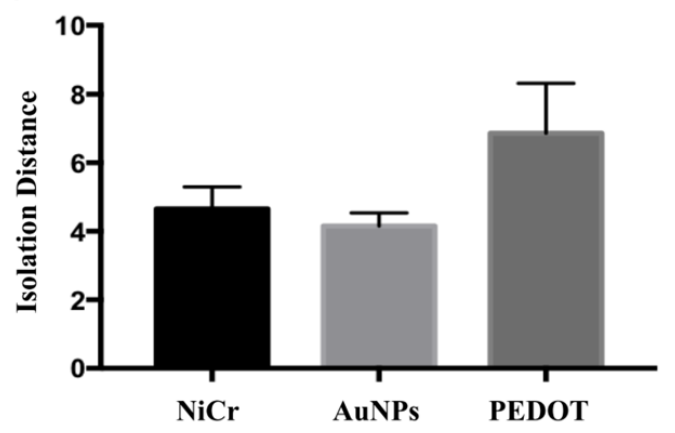

b)

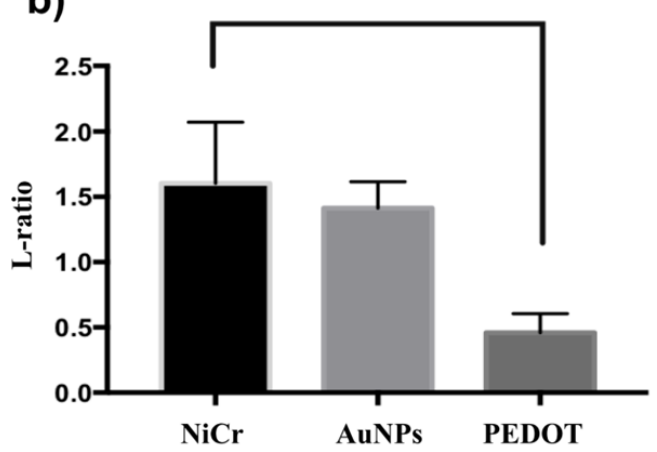

Fig. 6. a) Isolation distance of the bare $\mathrm{NiCr}(n=3)$, AuNPs-coated $(n=3)$ and AuNPs-PEDOT-coated ( $n=3)$ tetrodes; b) L-ratio values of the bare NiCr $(n=3)$, AuNPs-coated $(n=3)$ and AuNPs-PEDOT-coated $(n=3)$ tetrodes. The levels of statistical significance were indicated as follows ${ }^{*} p<0.01$. These values were calculated using a previously reported method [41].

Table 2. Average impedance at 1 kHz, L-ratio, ID values, SNR and RMSnoise of bare NiCr, AuNPs-coated and AuNPs-PEDOT-coated tetrodes

\begin{tabular}{lccc}
\hline & Bare NiCr & NiCr-AuNPs & NiCr-AuNPs-PEDOT \\
\hline Average impedance at $1 \mathrm{kHz}(\mathrm{M} \Omega)$ & $4.34 \pm 0.31$ & $0.33 \pm 0.04$ & $0.013 \pm 0.001$ \\
Average L-ratio & $1.603 \pm 0.47$ & $1.414 \pm 0.20$ & $0.46 \pm 0.14$ \\
Average isolation distance & $4.65 \pm 0.64$ & $4.16 \pm 0.38$ & $6.86 \pm 1.46$ \\
SNR $(\mathrm{dB})$ & $8.72 \pm 0.03$ & $8.90 \pm 0.25$ & $9.02 \pm 0.59$ \\
RMSnoise $(\mu \mathrm{V})$ & $44.9 \pm 0.001$ & $44.6 \pm 0.002$ & $44.8 \pm 0.004$ \\
\hline
\end{tabular}

All data are presented as the mean \pm SD.

L-ratio and Isolation distance (ID) value that can quantitatively measure the quality of a cluster which confirms the degree of signal isolation obtained from the intracellular recording data. As shown in Fig. 6, the AuNPs-PEDOT-coated tetrode significantly reduced the L-ratio of the spike signals of the AuNPs-PEDOTcoated tetrode was improved with those of the bare and AuNPscoated electrodes. These results demonstrate that the recording performance of the neural activity in the VPM could be improved due to the enhanced effective surface area of the modified electrode and could contribute to improve recording and cluster quality. The multiunit neural activity was increased in the AuNPsPEDOT-coated group compared to that in the bare NiCr group.

\section{DISCUSSION}

This paper demonstrated the effect of PEDOT and AuNPs on the surface of tetrodes, which improved the electrochemical properties and neural recording quality. The use of tetrodes can increase the probability of recording neural signals and identify neurons. Tetrodes are useful tool for neural recordings, and many studies have reported that coatings on the surfaces of tetrodes can efficiently improve the probability of detecting neural signals. Some studies have shown the beneficial effects of AuNPs and
PEDOT separately as separate coating materials [6, 16, 33, 48]. However, the aim of this study was to demonstrate the possibility of electroplating PEDOT additionally onto AuNPs-coated bare wires. Our results show that the application of this double coating (AuNPs-PEDOT) to a tetrode surface has the potential to improve the electrochemical properties and neural recording quality.

We found that the impedance value of AuNPs-PEDOT-coated tetrodes was significantly reduced by $4.3 \mathrm{M} \Omega$ to approximately $13 \mathrm{k} \Omega$, i.e., by approximately three orders of magnitude. The impedance values of the bare gold microelectrodes modified with PEDOT nanotubes decreased by approximately two orders of magnitude compared to those of bare gold electrodes [31]. The impedance magnitude of Ir microelectrodes after IrOx and PEDOT deposition decreased by approximately $404.5 \mathrm{k} \Omega$ to 23.3 $\mathrm{k} \Omega$ [22]. This result might indicate that the initial impedance value of the NiCr wire was relatively high, but the impedance value decreased greatly after the sequential electrodeposition of AuNPs and PEDOT, resulting in coated tetrodes that were more suitable as neural recording electrode. The CSCs of the modified tetrodes increased compared with those of the bare tetrode. It is reasonably assumed that electroplating PEDOT and AuNPs onto a tetrode effectively increases the surface area of the recording sites, which is directly correlated with the recording quality. Moreover, the 
AuNPs-PEDOT-coated tetrodes could transfer more charge than the bare NiCr wire. We demonstrated that the additional electroplating of PEDOT onto the AuNPs-coated electrode surface significantly reduced the impedance value and increased the cathodic storage capacity. Lowered impedance could be interpreted as the recording quality, and is derived from the SNR and noise values from in vivo recording. Furthermore, the modified tetrode tips could effectively isolate neural spikes compared with unmodified tetrodes, which can be obtained from L-ratio and ID values. Thus, reduced impedance of recording site might contribute to slightly increased SNR with reducing thermal noise and increase the resolvable number of neurons $[49,50]$. These results show that the modified-tetrode could effectively separate a single-unit neuron from the spikes.

Reducing the impedance value of an electrode is important, as this parameter can influence the suitability of an electrode for both brain recording and electrostimulation [35]. PEDOT was selected as a coating material for AuNPs- coated tetrodes due to its conductive properties, which can improve the contact at the electrode/ tissue interface. The application of a PEDOT coating to electrodes can significantly reduce the electrical impedance and charge injection in vitro with lowering charge transfer resistance $[35,48]$. Lowering the impedance of electrode is known to improve neural recording because low-impedance electrode could reduce thermal noise and minimize signal loss through shunt pathways $[49,50]$. Reduced impedance is contributed to lowering noise and increasing SNR, which can help recording quality. Increased SNR could contribute to increasing the number of resolvable neurons. The ability of recording various numbers of neuron could be represented by L-ratio and ID values in terms of sensitivity, which indicates the quality of clustering. Furthermore, PEDOT-coated tetrodes can be applied for use as electrostimulation electrodes and as CP electroplating materials [30, 45]. The electrical conductivity of a CP can increase with the doping process. Since dopants can play an important role in reducing the impedance value and can increase the CSCc, a comparison between PEDOT and various dopants as coating materials may be performed in future studies.

For long-term in vivo recording, electrodes must possess a high SNR, a high spatial resolution, a low impedance value and biocompatibility. It is difficult for traditional metal electrodes such as NiCr, Pt and Pt/Ir to satisfy these requirements. Therefore, biocompatible CPs are suitable if these polymers have a high conductivity, which is directly related to providing the electrode with a low impedance value. Additionally, highly stable materials are desired to ensure that they endure the implantation process. Among CPs, PEDOT has better electrochemical stability than that of PPy due to its structure [35]. Hence, PEDOT is a reasonably suitable coating material for long-term in vivo recording.

There are some limitations of this study. First, we performed the investigation using a limited number of materials. For a better comparison to demonstrate the abilities of the AuNPs-PEDOTcoated tetrodes, they need to be compared with other materials (e.g., tungsten, $\mathrm{Pt} / \mathrm{Ir}$ and $\mathrm{IrOx}$ ). Second, the surface areas of all tested tetrodes could not be accurately determined. Furthermore, $13-\mu \mathrm{m}$ bare $\mathrm{NiCr}$ wire insulated with polyimide was used by cutting with sharp scissors, but the exposed surface area can change depending on the cutting angle. In this paper, it was assumed that the cutting blade and the wire were exactly perpendicular to each other. In addition, the degrees of coating and surface roughness values were not mentioned, even though the electroplating experiments with AuNPs and PEDOT were carried out under the same conditions. To carry out a comparison to determine the characteristics of AuNPs-PEDOT-coated tetrodes, the surface roughness or amount of coating material should be discussed. Third, the longterm stability of the tetrodes must be considered for in vivo neural signal recording. We did not check the long-term stability of the AuNPs-PEDOT-coated tetrodes after a few weeks. To the best of our knowledge, PEDOT is well known as a material that can be used to improve the long-term performance of microfabricated devices [30]. This long-term stability for recording suggests that a new variant of PEDOT may be suitable for chronic neural application [28]. The use of the PEDOT materials proposed in this paper as an electrode coating is still far from being applicable to human cortical electrode recording at the current technology level. Further studies must test the long-term stability, economic efficiency and recording reliability of the modified tetrode. For in vivo recording, anesthetized rats were used to obtain the local field potential and spikes. All the experiments involving rats were conducted under anesthesia conditions, and the recording equipment was fixed in a Faraday cage to reduce the background noise. The in vivo recording results could change depending on the animal conditions, anesthesia states of rats and laboratory conditions. Furthermore, SNR could not replace all 'ideal' neural information from in vivo recording. SNR may not representative of all complex neuronal system, and evaluating neural electrode assumes that action potential arises from each single neuronal system, therefore it underestimates complex neural network. Due to small electrode size and completely uncontrolled background noise, it might have large RMS values rather than some reported RMS values [25, 45]. However, our study on the AuNPs-PEDOT-coated tetrodes involved with not only in vitro but also in vivo recordings to identify the electrode properties. Therefore, this paper demonstrates the potential of AuNPs-PEDOT-coated tetrodes.

In summary, the tips of NiCr tetrodes were modified with AuNPs 
and PEDOT via electrochemical deposition due to improvement of electrochemical properties and neural signal recording quality. We demonstrated that additional electroplating of PEDOT onto the AuNPs-coated surface of electrodes significantly reduced the impedance value and increased the cathodic storage capacity. Surface modification of the tetrodes allowed to detect multi neural cell recording due to the reduced impedance and increased cathodic storage capacity. PEDOT-coated tetrode recordings can aid in specific research applications, and the use of these modified tetrodes for multiple neuronal recordings can improve the understanding of complex brain signal activities in vivo.

\section{ACKNOWLEDGEMENTS}

This work was supported by the National Research Foundation of Korea (NRF ICT 2016H1D5A1908909).

\section{REFERENCES}

1. Buzsáki G (2004) Large-scale recording of neuronal ensembles. Nat Neurosci 7:446-451.

2. Schjetnan AG, Luczak A (2011) Recording large-scale neuronal ensembles with silicon probes in the anesthetized rat. J Vis Exp 3282.

3. Hetherington PA, Swindale NV (1999) Receptive field and orientation scatter studied by tetrode recordings in cat area 17. Vis Neurosci 16:637-652.

4. Tamura K, Ohashi Y, Tsubota T, Takeuchi D, Hirabayashi T, Yaguchi M, Matsuyama M, Sekine T, Miyashita Y (2012) A glass-coated tungsten microelectrode enclosing optical fibers for optogenetic exploration in primate deep brain structures. J Neurosci Methods 211:49-57.

5. McCarthy PT, Madangopal R, Otto KJ, Rao MP (2009) Titanium-based multi-channel, micro-electrode array for recording neural signals. Conf Proc IEEE Eng Med Biol Soc 2009:2062-2065.

6. Cui X, Martin DC (2003) Fuzzy gold electrodes for lowering impedance and improving adhesion with electrodeposited conducting polymer films. Sens Acutuators A Phys 103:384394.

7. Cheung KC (2007) Implantable microscale neural interfaces. Biomed Microdevices 9:923-938.

8. Baranauskas G, Maggiolini E, Castagnola E, Ansaldo A, Mazzoni A, Angotzi GN, Vato A, Ricci D, Panzeri S, Fadiga L (2011) Carbon nanotube composite coating of neural microelectrodes preferentially improves the multiunit signal-to-noise ratio. J Neural Eng 8:066013.
9. Robinson DA (1968) The electrical properties of metal microelectrodes. Proc IEEE 56:1065-1071.

10. Heim M, Rousseau L, Reculusa S, Urbanova V, Mazzocco C, Joucla S, Bouffier L, Vytras K, Bartlett P, Kuhn A, Yvert B (2012) Combined macro-/mesoporous microelectrode arrays for low-noise extracellular recording of neural networks. J Neurophysiol 108:1793-1803.

11. Seker E, Berdichevsky Y, Begley MR, Reed ML, Staley KJ, Yarmush ML (2010) The fabrication of low-impedance nanoporous gold multiple-electrode arrays for neural electrophysiology studies. Nanotechnology 21:125504.

12. Schmidt M, Falco A, Loch M, Lugli P, Scarpa G (2014) Spray coated indium-tin-oxide-free organic photodiodes with PEDOT: PSS anodes. AIP Adv 4:107132.

13. Marzouk SA, Ufer S, Buck RP, Johnson TA, Dunlap LA, Cascio WE (1998) Electrodeposited iridium oxide $\mathrm{pH}$ electrode for measurement of extracellular myocardial acidosis during acute ischemia. Anal Chem 70:5054-5061.

14. Negi S, Bhandari R, Rieth L, Solzbacher F (2010) In vitro comparison of sputtered iridium oxide and platinum-coated neural implantable microelectrode arrays. Biomed Mater 5:15007.

15. Cui X, Lee VA, Raphael Y, Wiler JA, Hetke JF, Anderson DJ, Martin DC (2001) Surface modification of neural recording electrodes with conducting polymer/biomolecule blends. J Biomed Mater Res 56:261-272.

16. Ferguson JE, Boldt C, Redish AD (2009) Creating lowimpedance tetrodes by electroplating with additives. Sens Actuators A Phys 156:388-393.

17. Blanche TJ, Spacek MA, Hetke JF, Swindale NV (2005) Polytrodes: high-density silicon electrode arrays for large-scale multiunit recording. J Neurophysiol 93:2987-3000.

18. Li X, Wang Lu, Fan Y, Feng Q, Cui F (2012) Biocompatibility and toxicity of nanoparticles and nanotubes. J Nanomater 2012:548389.

19. Kim YH, Kim GH, Kim AY, Han YH, Chung MA, Jung SD (2015) In vitro extracellular recording and stimulation performance of nanoporous gold-modified multi-electrode arrays. J Neural Eng 12:066029.

20. Zhang S, Wang N, Yu H, Niu Y, Sun C (2005) Covalent attachment of glucose oxidase to an Au electrode modified with gold nanoparticles for use as glucose biosensor. Bioelectrochemistry 67:15-22.

21. Choi JH, Kim HS, Choi JW, Hong JW, Kim YK, Oh BK (2013) A novel Au-nanoparticle biosensor for the rapid and simple detection of PSA using a sequence-specific peptide cleavage reaction. Biosens Bioelectron 49:415-419. 
22. Wilks SJ, Richardson-Burns SM, Hendricks JL, Martin DC, Otto KJ (2009) Poly(3,4-ethylenedioxythiophene) as a microneural interface material for electrostimulation. Front Neuroeng 2:7.

23. Richardson-Burns SM, Hendricks JL, Foster B, Povlich LK, Kim DH, Martin DC (2007) Polymerization of the conducting polymer poly (3,4-ethylenedioxythiophene) (PEDOT) around living neural cells. Biomaterials 28:1539-1552.

24. Abidian MR, Corey JM, Kipke DR, Martin DC (2010) Conducting-polymer nanotubes improve electrical properties, mechanical adhesion, neural attachment, and neurite outgrowth of neural electrodes. Small 6:421-429.

25. Ludwig KA, Uram JD, Yang J, Martin DC, Kipke DR (2006) Chronic neural recordings using silicon microelectrode arrays electrochemically deposited with a poly(3,4-ethylenedioxythiophene) (PEDOT) film. J Neural Eng 3:59-70.

26. Cogan SF (2008) Neural stimulation and recording electrodes. Annu Rev Biomed Eng 10:275-309.

27. Keefer EW, Botterman BR, Romero MI, Rossi AF, Gross GW (2008) Carbon nanotube coating improves neuronal recordings. Nat Nanotechnol 3:434-439.

28. Aregueta-Robles UA, Woolley AJ, Poole-Warren LA, Lovell NH, Green RA (2014) Organic electrode coatings for nextgeneration neural interfaces. Front Neuroeng 7:15.

29. Cui X, Wiler J, Dzaman M, Altschuler RA, Martin DC (2003) In vivo studies of polypyrrole/peptide coated neural probes. Biomaterials 24:777-787.

30. Charkhkar H, Knaack GL, McHail DG, Mandal HS, Peixoto N, Rubinson JF, Dumas TC, Pancrazio JJ (2016) Chronic intracortical neural recordings using microelectrode arrays coated with PEDOT-TFB. Acta Biomater 32:57-67.

31. Abidian MR, Martin DC (2008) Experimental and theoretical characterization of implantable neural microelectrodes modified with conducting polymer nanotubes. Biomaterials 29:1273-1283.

32. Abidian MR, Ludwig KA, Marzullo TC, Martin DC, Kipke DR (2009) Interfacing conducting polymer nanotubes with the central nervous system: chronic neural recording using poly(3,4-ethylenedioxythiophene) nanotubes. Adv Mater 21:3764-3770.

33. Ludwig KA, Langhals NB, Joseph MD, Richardson-Burns SM, Hendricks JL, Kipke DR (2011) Poly(3,4-ethylenedioxythiophene) (PEDOT) polymer coatings facilitate smaller neural recording electrodes. J Neural Eng 8:014001.

34. Venkatraman S, Hendricks J, King ZA, Sereno AJ, RichardsonBurns S, Martin D, Carmena JM (2011) In vitro and in vivo evaluation of PEDOT microelectrodes for neural stimulation and recording. IEEE Trans Neural Syst Rehabil Eng 19:307316.

35. Cui X, Martin DC (2003) Electrochemical deposition and characterization of poly(3,4-ethylenedioxythiophene) on neural microelectrode arrays. Sens Actuators B Chem 89:92102.

36. Balint R, Cassidy NJ, Cartmell SH (2014) Conductive polymers: towards a smart biomaterial for tissue engineering. Acta Biomater 10:2341-2353.

37. Peramo A, Urbanchek MG, Spanninga SA, Povlich LK, Cederna P, Martin DC (2008) In situ polymerization of a conductive polymer in acellular muscle tissue constructs. Tissue Eng Part A 14:423-432.

38. Gray CM, Maldonado PE, Wilson M, McNaughton B (1995) Tetrodes markedly improve the reliability and yield of multiple single-unit isolation from multi-unit recordings in cat striate cortex. J Neurosci Methods 63:43-54.

39. Wilson MA, McNaughton BL (1993) Dynamics of the hippocampal ensemble code for space. Science 261:1055-1058.

40. Ferguson JE, Boldt C, Redish AD (2009) Creating lowimpedance tetrodes by electroplating with additives. Sens Actuators A Phys 156:388-393.

41. Schmitzer-Torbert N, Jackson J, Henze D, Harris K, Redish AD (2005) Quantitative measures of cluster quality for use in extracellular recordings. Neuroscience 131:1-11.

42. Harris KD, Hirase H, Leinekugel X, Henze DA, Buzsáki G (2001) Temporal interaction between single spikes and complex spike bursts in hippocampal pyramidal cells. Neuron 32:141-149.

43. Quiroga RQ, Nadasdy Z, Ben-Shaul Y (2004) Unsupervised spike detection and sorting with wavelets and superparamagnetic clustering. Neural Comput 16:1661-1687.

44. Rey HG, Pedreira C, Quian Quiroga R (2015) Past, present and future of spike sorting techniques. Brain Res Bull $119 \mathrm{Pt}$ B:106-117.

45. Du ZJ, Luo X, Weaver C, Cui XT (2015) Poly (3, 4-ethylenedioxythiophene)-ionic liquid coating improves neural recording and stimulation functionality of MEAs. J Mater Chem C Mater 3:6515-6524.

46. Franks W, Schenker I, Schmutz P, Hierlemann A (2005) Impedance characterization and modeling of electrodes for biomedical applications. IEEE Trans Biomed Eng 52:1295-1302.

47. Lu Y, Li T, Zhao X, Li M, Cao Y, Yang H, Duan YY (2010) Electrodeposited polypyrrole/carbon nanotubes composite films electrodes for neural interfaces. Biomaterials 31:5169-5181.

48. Xiao Y, Cui X, Martin DC (2004) Electrochemical polymerization and properties of PEDOT/S-EDOT on neural micro- 
electrode arrays. J Electroanal Chem 573:43-48.

49. Kovacs GTA (1994) Introduction to the theory, design, and modeling of thin-film microelectrodes for neural interfaces. In: Enabling technologies for cultured neural networks (Stenger DA, McKenna T, eds), pp 121-165. Academic Pub- lishers, NewYork, NY.

50. Humphrey DR, Schmidt EM (1990) Extracellular single-unit recording methods. In: Neurophysiological techniques: applications to neural systems (Boulton AA, Baker GB, Vanderwolf CH, eds), pp 1-64. Springer, Clifton, NJ. 